
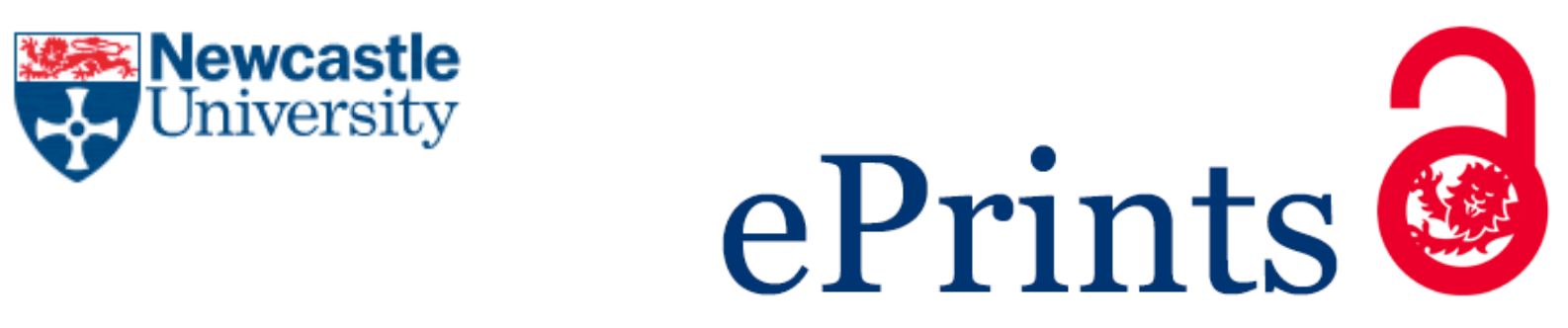

Aygueler MF, Weber MD, Puscher BMD, Medina DD, Docampo P, Costa RD. Light-Emitting Electrochemical Cells Based on Hybrid Lead Halide Perovskite Nanoparticles.

The Journal of Physical Chemistry C 2015, 119(21), 12047-12054.

\title{
Copyright:
}

This document is the Accepted Manuscript version of a Published Work that appeared in final form in the Journal of Physical Chemistry C, copyright (c) American Chemical Society after peer review and technical editing by the publisher. To access the final edited and published work see http://dx.doi.org/10.1021/acs.jpcc.5b02959

Date deposited:

$31 / 05 / 2017$

Embargo release date:

15 April 2016 


\section{Light-Emitting Electrochemical Cells Based on Hybrid Lead Halide Perovskite Nanoparticles}

Meltem F. Aygüler, ${ }^{1, \dot{t}}$ Michael D. Weber, ${ }^{2, \dot{t}}$ Bianka M. D. Puscher, ${ }^{2}$ Dana D. Medina, ${ }^{1}$ Pablo Docampo, ${ }^{1, *}$ Rubén D. Costa, ${ }^{2, *}$

${ }^{1}$ Department of Chemistry and Center for Nanoscience, University of Munich (LMU), Butenandtstr. 11, D-81377 Munich, Germany

${ }^{2}$ Department of Chemistry \& Pharmacy, University of Erlangen-Nuremberg (FAU), Egerlandstr. 3, D-91058 Erlangen, Germany 


\begin{abstract}
Methylammonium lead bromide $\left(\mathrm{MAPbBr}_{3}\right)$ perovskite nanoparticles (NPs) have been recently proposed as a new material for light-emitting diodes, as well as a new paradigm to elucidate the operational mechanism in perovskite solar cells. Here, we have expanded the synthesis concept to fabricate NPs based on formamidinium lead bromide $\left(\mathrm{FAPbBr}_{3}\right)$. Importantly, we have demonstrated that the photophysical features of this novel material can be easily tuned by exchanging the organic cation, achieving lower radiative bimolecular recombination rate for $\mathrm{FAPbBr}_{3}$ NPs. Additionally, we report for the first time light-emitting electrochemical cells (LECs) based on perovskite NPs by an easily up-scalable spray-coating technique. Stable luminance of $1-2 \mathrm{~cd} / \mathrm{m}^{2}$ at low driving currents was achieved for both types of materials. Overall, this work opens a new avenue of research into the field of organic-inorganic metal halide nanoparticles bearing different alkyl ammonium groups and their application in the developing field of thin-film lighting devices.
\end{abstract}

\title{
INTRODUCTION
}

Light-emitting electrochemical cells (LECs) have recently emerged as a potential alternative to organic light-emitting diodes (OLEDs) due to the use of air-stable injection layers and/or electrodes, as well as its simple device architecture consisting of a single layer sandwiched between two electrodes $-e . g$, indium tin-oxide (ITO) and aluminum. ${ }^{1-4}$ In addition, they can operate at very low voltages, yielding highly efficient devices, which can be easily fabricated under ambient conditions on any kind of substrate. ${ }^{3}$

In LECs, the active layer typically consists of either a mixture of a light-emitting polymer, an ion conducting polymer and an inorganic salt or only one ionic transition-metal complex 
(iTMC). ${ }^{1-3}$ However, other luminescent materials can also be easily incorporated. ${ }^{5-8}$ For instance, Bader et al. reported on yellow and orange LECs incorporating a blend of $\mathrm{CdSe} / \mathrm{ZnS}$ quantum dots (QDs), a PF/PPV copolymer - poly[(9,9-dioctyl-2,7-divinylene-fluorenylene)-alt-co-\{2meth-oxy-5-(2-ethyl-hexyloxy)-1,4 phenylene\}], and the polymerizable ionic liquid allyltrioctylammonium allylsulfonate (ATOAAS) as the source of counter ions. ${ }^{7}$ Recently, Qian et al. expanded this concept to include blue, green, red, and white, conventional and flexible QDLECs. ${ }^{8}$

The major limitation of inorganic QDs in lighting applications is the significant reduction of the photoluminescence quantum yields (PLQYs) after ligand exchange, a step that is necessary for their application in devices. ${ }^{8}$ Additionally, all the developed QDs exhibit significant subbandgap defects, which effectively limit the maximum device performance achievable. ${ }^{9}$ This issue is, however, not present in the newly developed organic-inorganic metal halide perovskites, where grain boundaries and surface defects do not generate deep trap states within the bandgap. ${ }^{10-11}$ For this reason, the application of these materials in lighting applications is expected to enable the fabrication of highly efficient and low-cost devices.

Previously, electroluminescence (EL) from perovskite films was only achieved by using either low temperatures $(77-110 \mathrm{~K})$ or by introducing light-emitting organic compounds into the crystal lattice. ${ }^{12-15} \mathrm{EL}$ from bulk films at room temperature was recently achieved by Tan et al. ${ }^{16}$ and Kim et al. ${ }^{17}$ while Schmidt et al. ${ }^{18}$ reported on the first synthesis of $\mathrm{MAPbBr}_{3}$ perovskite NPs, showing the possibility to use them as light-emitting materials in OLEDs. This result represents an important contribution to the field, since the preparation of perovskite NPs is the first step to facilitate their implementation in new thin film device architectures by blending them with other electroactive materials. 
Here, we highlight two major aspects of our current work. Firstly, we have expanded the fundamental knowledge regarding the synthesis of new hybrid metal halide perovskite NPs, showing that the photophysical features of this novel material can be tuned by exchanging the organic cation group to formamidinium (FA). Secondly, we have taken advantage of the possibility to stabilize this novel material in solution to fabricate the first $\mathrm{MAPbBr}_{3^{-}}$and $\mathrm{FAPbBr}_{3}$-based LECs featuring a luminance of around $1-2 \mathrm{~cd} / \mathrm{m}^{2}$ at low driving currents by developing a deposition protocol based on spray-coating, bringing this technology closer to large-scale applications.

\section{EXPERIMENTAL SECTION}

\section{Synthesis of precursors:}

Methylammonium bromide was prepared by following published procedure. ${ }^{19}$ In short, $24 \mathrm{ml}$ of methylamine solution (33 wt. \% in ethanol) was diluted with $100 \mathrm{ml}$ of absolute ethanol in a $250 \mathrm{~mL}$ round bottom flask. Under constant stirring, $8 \mathrm{ml}$ of an aqueous solution of hydrobromic acid (48 wt. \%) was added to the flask. After stirring for one hour at room temperature, the solvent was evaporated. The obtained white solid was washed with dry diethyl ether and finally recrystallized from ethanol.

Formamidinium bromide was synthesized following a known procedure. ${ }^{19}$ Formamidinium acetate was dissolved in a double molar excess of aqueous $\mathrm{HBr}(48$ wt. \%), and the mixture was stirred at $50{ }^{\circ} \mathrm{C}$ for 60 minutes. The solvent and remaining $\mathrm{HBr}$ were removed by rotary evaporation. Slightly yellow powder was washed with dry diethyl ether. Lastly, recrystallization from ethanol led to the white crystalline product.

\section{Synthesis of octylammonium bromide:}


Octylamine $(24.79 \mathrm{ml}, 0.15 \mathrm{~mol})$ was added to $200 \mathrm{ml}$ of absolute ethanol in a $500 \mathrm{ml}$ round bottom flask. Hydrobromic acid (5 M, $30 \mathrm{ml}, 0.15 \mathrm{~mol})$ was added to the solution under constant stirring. After a reaction time of one hour at room temperature, the solvent was removed by rotary evaporation. The product was washed with diethyl ether until the powder became white. Then, octylammonium bromide was recrystallized from 2-propanol.

\section{Synthesis of nanoparticles and characterization details}

\section{Synthesis of $\mathrm{MAPbBr}_{3}$ nanoparticles}

A solution of oleic acid $(850 \mathrm{mg}, 3 \mathrm{mmol})$ in $20 \mathrm{ml}$ of octadecene was stirred and heated at $80{ }^{\circ} \mathrm{C}$ in a $100 \mathrm{ml}$ round bottom flask under $\mathrm{N}_{2}$ atmosphere. Then, capping agent, octylammonium bromide (126 mg, $0.6 \mathrm{mmol})$ was added to the flask and the solution became cloudy. Lead (II) bromide (367 mg, $1 \mathrm{mmol}$ dissolved in $1 \mathrm{ml}$ of anhydrous DMF at $70{ }^{\circ} \mathrm{C}$ ) was added to the reaction vessel and a clear and colorless solution was formed. Subsequent and dropwise addition of methylammonium bromide (44 mg, $0.4 \mathrm{mmol}$ dissolved in $1 \mathrm{ml}$ of anhydrous DMF) produced a yellow dispersion. Lastly, nanoparticles were immediately precipitated by addition of anhydrous THF $(25 \mathrm{ml})$. The suspension was centrifuged at $7000 \mathrm{rpm}$ for 10 minutes. The NPs sedimentation and the solution were removed. Subsequently the NPs were redispersed in THF and centrifuge for three more times in order to remove remaining oleic acid and octadecene. Nanoparticles were dried in air to obtain orange powder and finally placed into vial and were redispersed in anhydrous THF.

\section{Synthesis of $\mathrm{FAPbBr}_{3}$ nanoparticles}

A solution of oleic acid (170 mg, $0.6 \mathrm{mmol})$ in $4 \mathrm{ml}$ of octadecene was stirred and heated at $80{ }^{\circ} \mathrm{C}$ in a $20 \mathrm{ml}$ vial in air. Then, capping agent, octylammonium bromide $(25.2 \mathrm{mg}, 0.12 \mathrm{mmol})$ was added to the vial and the solution became cloudy. Lead (II) bromide (73.4 mg, $0.08 \mathrm{mmol}$ 
dissolved in $200 \mu \mathrm{l}$ of anhydrous DMF at $70^{\circ} \mathrm{C}$ ) was added to the reaction vessel and clear and colorless solution was formed. Subsequent and dropwise addition of formamidinium bromide (10 mg, $0.08 \mathrm{mmol}$ dissolved in $200 \mu \mathrm{l}$ of anhydrous DMF) produced a yellow dispersion. Lastly, nanoparticles were immediately precipitated by addition of anhydrous THF (15 ml). The suspension was centrifuged at $7000 \mathrm{rpm}$ for 10 minutes. The NPs sedimentation and the solution were removed. Subsequently the NPs were redispersed in THF and centrifuge for three more times in order to remove remaining oleic acid and octadecene. Nanoparticles were dried in air to obtain orange powder and finally placed into vial and were redispersed in anhydrous THF.

\section{Characterization details of nanoparticles}

The powder XRD measurements were performed on a Huber Imaging Plate Guinier Diffractometer G670 (Cu-Ka1-radiation). Steady-state absorption spectra were measured with a Lambda $1050 \mathrm{UV} /$ Vis spectrophotometer (Perkin Elmer) using an integrating sphere. Steady state efficiency and time resolved PL measurements were performed with a Fluotime 300 Spectrofluorometer (Picoquant $\mathrm{GmbH}$ ). The excitation wavelength was fixed at $405 \mathrm{~nm}$. The emission for time resolved measurements was monitored at the maximum intensity of the steady state photo emission. ${ }^{1} \mathrm{H}-\mathrm{NMR}$ spectra were acquired at $25.8^{\circ} \mathrm{C}$ using a Bruker Avance III HD spectrometer with a $400 \mathrm{MHz}$ magnet $(9.4 \mathrm{~T})$. The chemical shifts are reported in ppm relative to tetramethylsilane (TMS). TEM measurements were performed with JEM-2011 (JEOL) equipped with $\mathrm{LaB}_{6}$ operated at $200 \mathrm{kV}$ and EDS (EDAX) detector. The PLQYs were measured using a Fluoromax-4 photometer with a quanta-phi integrating sphere upon excitation of $400 \mathrm{~nm}$. The films were prepared onto quartz slides. The samples were excited with Fluotime 300 Spectrofluorometer (Picoquant $\mathrm{GmbH}$ ) with a diode laser at $405 \mathrm{~nm}$ with $800 \mathrm{ps}$ pulse length and at different excitation fluences. AFM characterization was performed under ambient conditions 
using SPM Nanoscope Illa multimode working in tapping mode with a HQ:NSC15/AL BS tip ( $\mu$ Masch) at a working frequency of $\sim 325 \mathrm{KHz}$.

\section{Device fabrication and characterization details}

Double layers LECs were fabricated. The first layer consists of $100 \mathrm{~nm}$ of poly(3,4ethylenedioxythiophene):poly(styrenesulfonate) (PEDOT:PSS), which is used to flatten the transparent conducting electrode - i.e., indium tin oxide, ITO, while the second layer with a thickness ranging from 60-120 nm was the luminescent layer. All materials were purchased from Sigma Aldrich and used as received. The devices were prepared as follows. ITO coated glass plates were patterned by conventional photolithography (Naranjo Substrates). The substrates were cleaned by using sequential ultrasonic baths in detergent, water, and 2-propanol solvents. After drying, the substrates were placed in an UV-ozone cleaner (Jelight 42-220) for 10 min. A PEDOT:PSS layer was doctor-bladed onto the ITO-glass substrate to increase the device preparation yield (400 $\mu \mathrm{m}$ substrate distance and a speed of $10 \mathrm{~mm} / \mathrm{s})$. The luminescent layer comprised perovskite NPs and the polyelectrolyte matrix - i.e., the optimized $\mathrm{LiCF}_{3} \mathrm{SO}_{3}$ and trimethylolpropane ethoxylate (TMPE) with the mass ratio of $0.3: 1$ dissolved in THF, respectively. The electrolyte mixture was prepared via stirring for five hours followed by ultrasonication for $20 \mathrm{~min}$ after the perovskite NPs were added $(1-2 \mathrm{mg} / \mathrm{mL})$. We found this mixture as the most appropriated for spray-coating. Higher amounts tend to quickly agglomerate, hampering the deposition procedure. Finally, the solution was filtered using polytetrafluorethylene membrane filters with a pore size of $0.45 \mu \mathrm{m}$. The process was repeated until an orange stable suspension was achieved. The active layer was prepared by using spraypyrolysis technique - i.e., Ar flow at 1.5 atm and $90{ }^{\circ} \mathrm{C}$. These conditions resulted in homogenous thin films with a thickness ranging from 60 to $120 \mathrm{~nm}$ and a roughness of less than 
$5 \%$, having no apparent optical defects. The latter was determined using a Bruker 'DektakxT' profilometer. Once the active layer was deposited, the samples were transferred into an inert atmosphere glovebox $\left(<0.1 \mathrm{ppm} \mathrm{O}_{2}\right.$ and $\mathrm{H}_{2} \mathrm{O}$, Innovative Technology). Aluminum cathode electrode $(90 \mathrm{~nm})$ was thermally evaporated using a shadow mask under high vacuum $\left(<1 \times 10^{-6}\right.$ mbar) using an Angstrom Covap evaporator integrated into the inert atmosphere glovebox. Time dependence of luminance, voltage, and current was measured by applying constant and/or pulsed voltage and current by monitoring the desired parameters simultaneously by using an Avantes spectrophotometer (Avaspec-ULS2048LTEC) calibrated with a white LED in conjunction with a Newport (1916-c) optical power meter equipped with a calibrated silicon diode and Botest OLT OLED Lifetime-Test System. Electroluminescence spectrum was recorded using the above mentioned spectrophotometer.

\section{RESULTS AND DISCUSSION}

Both $\mathrm{MAPbBr}_{3}$ and $\mathrm{FAPbBr}_{3}$ NPs were prepared through the addition of octylammonium bromide in a warm solution of oleic acid in octadecene followed by lead bromide and either methylammonium or formamidinium bromide. ${ }^{18}$ Here, we have found that the dropwise addition of the solution containing the organic cation is crucial to obtain pure compounds. Additionally, we have employed THF as the precipitating agent in order to compatibilize the NP solution with the electrolyte solution necessary for device deposition. This procedure results in a yelloworange colloidal solution of highly crystalline perovskite nanoparticles - Experimental Section for more details. The addition of a long chain alkyl ammonium cation was essential to limit the perovskite crystal growth, as reactions prepared without this capping agent resulted in micronsized crystals. ${ }^{18}$ 
Both synthesis protocols yielded highly crystalline perovskite NPs, which were characterized by powder X-ray diffraction (PXRD) in order to ascertain their crystal structure - Figure 1 and Table S1. The observed diffraction patterns obtained at room temperature correspond to the cubic crystal phase with lattice constants of 5.939(2) and 6.000(2) $\AA$ (both of a space group Pm3m) for $\mathrm{MAPbBr}_{3}$ and $\mathrm{FAPbBr}_{3} \mathrm{NPs}$, respectively; consistent with previous reports ${ }^{18-20}$ and their bulk counterparts (Figure S1a and b). Additionally, both NPs were characterized by ${ }^{1} \mathrm{H}-\mathrm{NMR}$ as shown in Figure $\mathrm{S} 2$ in the Supporting Information. The spectrum of $\mathrm{MAPbBr}_{3}$ NPs (Figure S2a) shows the peaks of both perovskite compound (2.38 and $7.55 \mathrm{ppm})$ and capping agent, octylammonium bromide - 0.87, 1.27, 1.52, 2.77 and $7.64 \mathrm{ppm}$. Figure S2b shows the ${ }^{1} \mathrm{H}-\mathrm{NMR}$ spectrum of $\mathrm{FAPbBr}_{3} \mathrm{NPs}$. The peaks at 9.02, 8.71, and $7.88 \mathrm{ppm}$ belong to perovskite material as seen in the inset graph, whereas the rest of the peaks show octylammonium bromide.

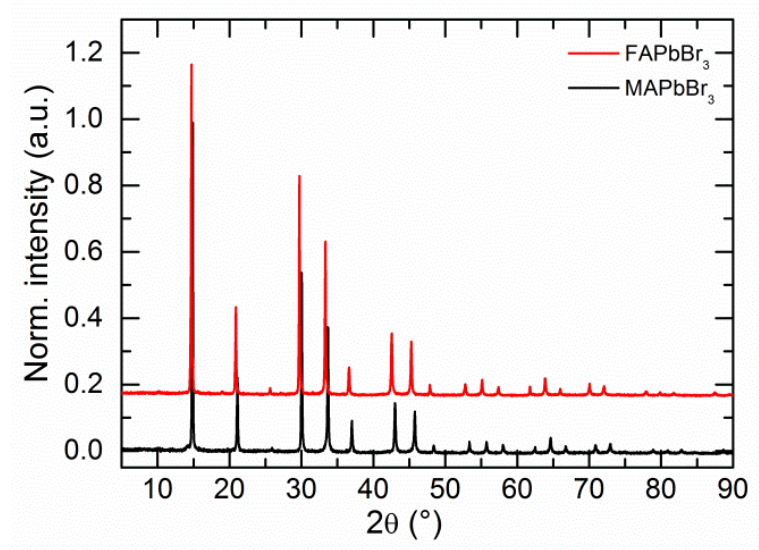

Figure 1. Powder X-ray diffraction pattern of both $\mathrm{MAPbBr}_{3}$ (black) and $\mathrm{FAPbBr}_{3}$ (red) NPs.

Scherrer-peak width analysis yielded crystallite sizes after solvent removal ranging from 50 to $90 \mathrm{~nm}$ - Figure S3. The size of the NPs was further confirmed by transmission electron microscopy (TEM). TEM images showed that the size distribution is not homogeneous since 
there are big aggregates as well as small-sized NPs $(<10 \mathrm{~nm})$ for both compositions - Figure S4 and S5.

The photophysical features of $\mathrm{MAPbBr}_{3}$ and $\mathrm{FAPbBr}_{3} \mathrm{NPs}$ were investigated by steady-state absorption and photoluminescence (PL) spectroscopy in solution - Figure 2. In particular, the absorption spectra of the $\mathrm{FAPbBr}_{3}$ colloidal solutions exhibit a narrow band at $542 \mathrm{~nm}$, which is $13 \mathrm{~nm}$ red-shifted compared to that of $\mathrm{MAPbBr}_{3}$ suspensions - Figure 2a. This is attributed mainly to the reduction of the optical band gap of $\mathrm{FAPbBr}_{3}$ NPs due to the larger unit cell. ${ }^{21}$ Indeed, the optical band gaps of $\mathrm{MAPbBr}_{3}$ and $\mathrm{FAPbBr}_{3} \mathrm{NPs}$ were found to be $2.24 \mathrm{eV}$ and 2.14 $\mathrm{eV}$ as estimated by Tauc plots - Figure S6. More interesting is, however, the direct comparison of the PL features between both types of perovskite NPs - Figure $2 \mathrm{~b}$. Both materials exhibit a near band edge emission with a narrow full width at the half-maximum (FWHM) of approximately $19 \mathrm{~nm}$. This corresponds to features noted for bulk-like films, ${ }^{19}$ indicating the high quality and crystallinity of the prepared NPs. The exchange of MA to FA results in a redshifted emission spectrum - i.e., going from 532 to $545 \mathrm{~nm}$ for $\mathrm{MAPbBr}_{3}$ and $\mathrm{FAPbBr}_{3} \mathrm{NPs}$, respectively.

a)

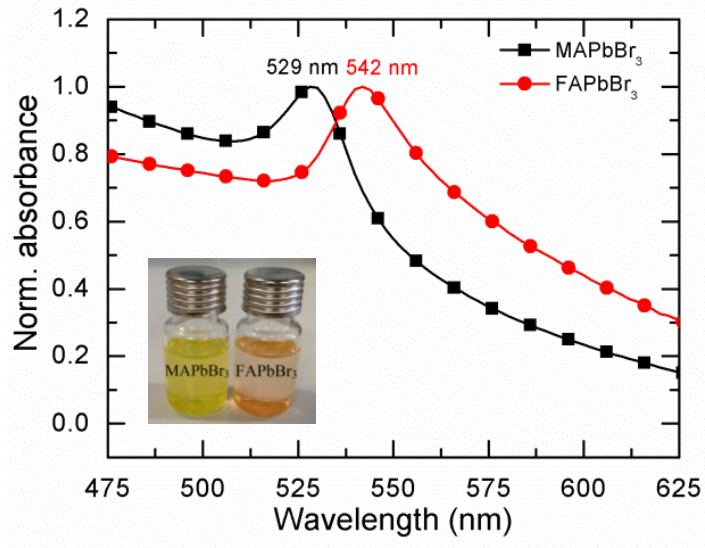

b)

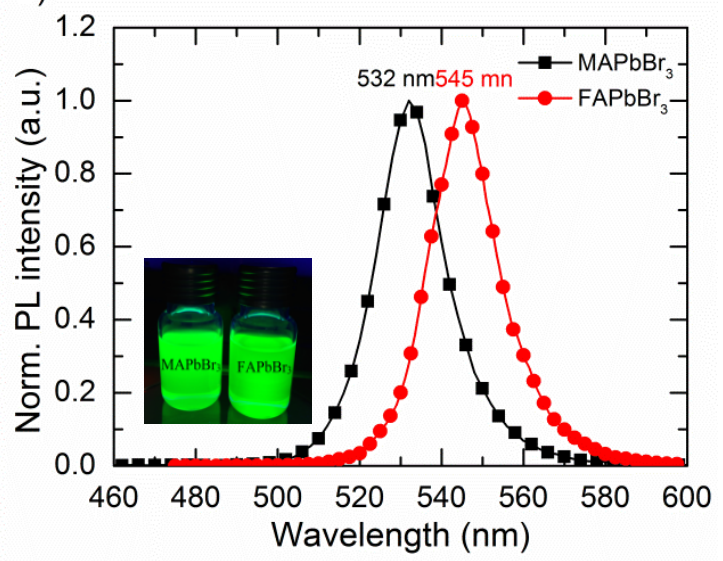




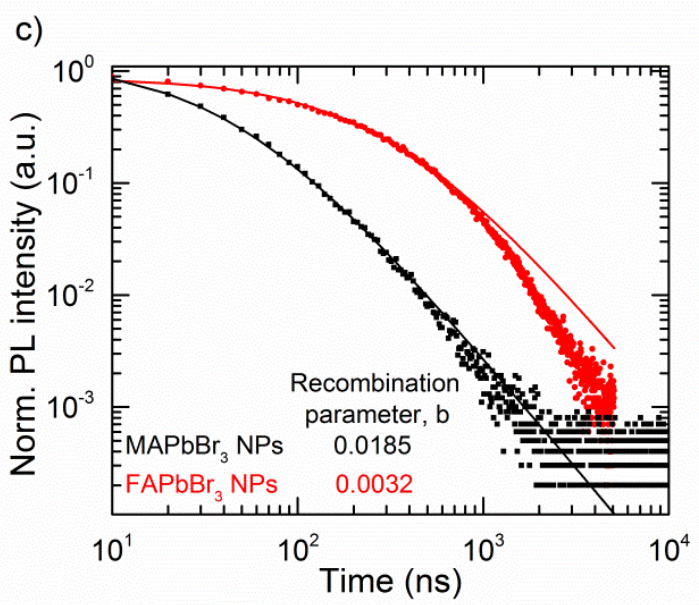

Figure 2. (a) Normalized absorption and (b) photoluminescence spectra at $403 \mathrm{~nm}$ excitation wavelength and $\sim 0.3 \mu \mathrm{J} / \mathrm{cm}^{2}$ pump fluence of $\mathrm{MAPbBr}_{3}$ (black squares) and $\mathrm{FAPbBr}_{3}$ (red circles) NPs in THF. Photographs of the solutions in THF are shown under ambient light (a) and $365 \mathrm{~nm}$ excitation (b). (c) Time-resolved PL decay for $\mathrm{MAPbBr}_{3}$ (black squares) and $\mathrm{FAPbBr}_{3}$ (red circles). Solid lines in time resolved PL decay curves are fits to the data with a simple bimolecular recombination model.

In order to further examine the differences in their photophysical behavior, the NPs were characterized in solution via time-resolved PL - Figure 2c. The obtained data was fit by a simple bimolecular recombination model: ${ }^{10,22-23}$

$$
f(t)=\frac{1}{a+b t}
$$

where $a$ is related to the charge density at $t=0$, and $b$ is the recombination parameter. The fittings suggest that the decay of the PL arises from bimolecular recombination of free charges, similar to that observed in perovskite films. ${ }^{10}$ Since both NP solutions absorbed light similarly at the excitation wavelength, and assuming that early time charge generation is similar for both materials, we can directly compare the fitted recombination parameters. The radiative bimolecular recombination rate of $\mathrm{FAPbBr}_{3} \mathrm{NPs}$ is approximately six times slower than 
$\mathrm{MAPbBr}_{3} \mathrm{NPs}$ - Figure 2c. While slow recombination dynamics are beneficial for solar cell operation, ${ }^{19}$ the opposite is true for light emitting devices since a reduction of the PL quantum yield (PLQY) is expected. To confirm this, the PLQYs in solution were determined with an integrating sphere. In line with the bimolecular recombination rate results, $\mathrm{MAPbBr}_{3}$ and $\mathrm{FAPbBr}_{3} \mathrm{NPs}$ exhibited PLQYs of approximately $15 \%$ and $5 \%$, respectively. It is important to point out that, similar to that noted by other groups,${ }^{18}$ the accuracy of the PLQYs is subjected to, the intrinsic scattering process of the suspensions, and the self-absorption due to the very small Stokes shift. We have additionally performed a pump fluence assay on both types of NPs as shown in Figure S7 in the Supporting Information. Here we observe that, at higher pump fluences, the PL response becomes sublinear, further confirming the radiative bimolecular recombination mechanism.

As a final step, we took advantage of the excellent stability of the perovskite NPs in solution to prepare the first perovskite-based LEC devices. To this end, an electrolyte matrix comprised of $\mathrm{LiCF}_{3} \mathrm{SO}_{3}$ and trimethylolpropane ethoxylate (TMPE) was added to the colloidal suspensions of the perovskite NPs and immediately filtered. The mixture of NPs and electrolyte matrix was spray-coated onto an ITO substrate which was modified by a $100 \mathrm{~nm}$ thickness of poly(3,4ethylenedioxythiophene) polystyrene sulfonate (PEDOT:PSS). In this work, active layers with two different thicknesses of approximately $100-120$ and 50-70 nm were prepared. To finalize the devices, aluminum was evaporated as a top cathode with a thickness of $90 \mathrm{~nm}$ - SI for more details. A schematic structure of this device is shown in Figure 4a.

One of the most difficult challenges when utilizing easily up-scalable techniques like spraycoating is the achievement of smooth and homogeneous films without degradation of the active material during film preparation. ${ }^{24-25}$ To ensure this, the morphological and spectroscopic 
features of the active layers were examined by means of alpha-step profilometry and atomic force microscopy (AFM), as well as steady-state spectroscopy, respectively. AFM confirms that the films show an homogenous coverage with no particular aggregation or phase separation features and all give a similar root-mean-square (RMS) roughness of around 5-14 nm - Figure 3. Furthermore, it was found that the addition of the matrix does not impact the PL emission maxima nor the PL decay dynamics compared to bare NPs films - Figure S8 and Figure S9.
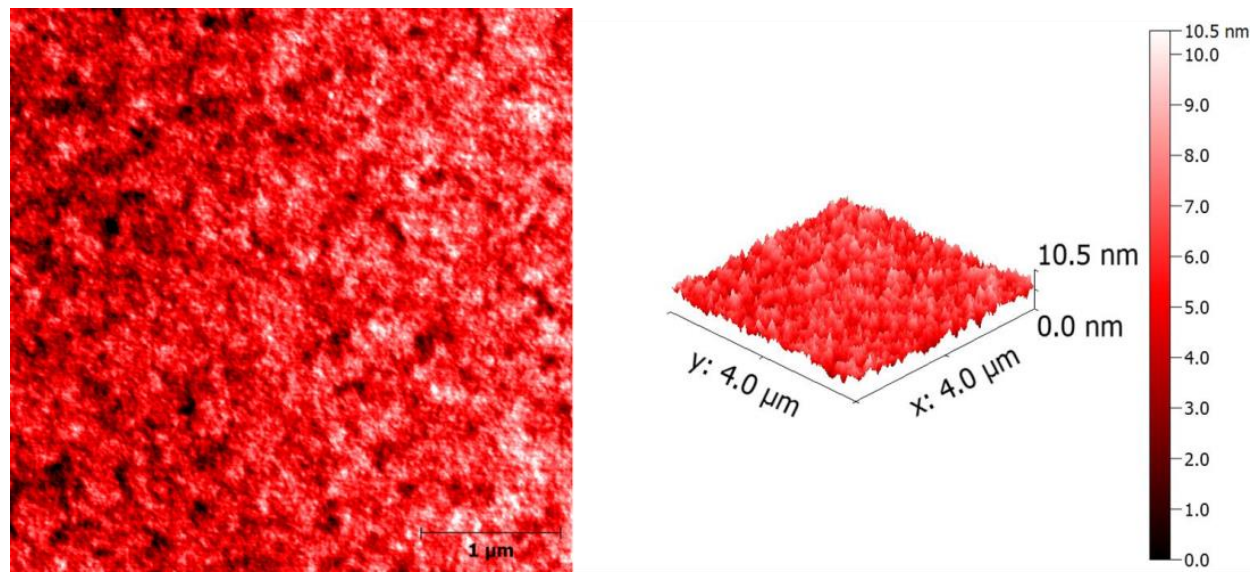

Figure 3. AFM top view image and 3D profile of perovskite NPs films used for device fabrication.

Figure $4 \mathrm{~b}$ and S10 depict the luminance-current density versus applied voltage (LIV) of the perovskite-based LECs. The LIV features of devices, which do not incorporate an electrolyte matrix, show an injection voltage of around 15-16 V independently of the type of perovskite NPs, but no luminance was detected, even when constant and pulsed voltage and/or current driving schemes or current LIVs were applied. Upon the addition of the electrolyte matrix, the devices feature injection voltages of around 11-12 V (at J $>0.1 \mathrm{~mA} / \mathrm{cm}^{2}$ ) and a maximum brightness of $1.3 \mathrm{~cd} / \mathrm{m}^{2}-$ Figure $4 \mathrm{~b}$. In a qualitative way, this finding clearly demonstrates the relevant role of the electrolyte matrix in the device behavior. 
a)

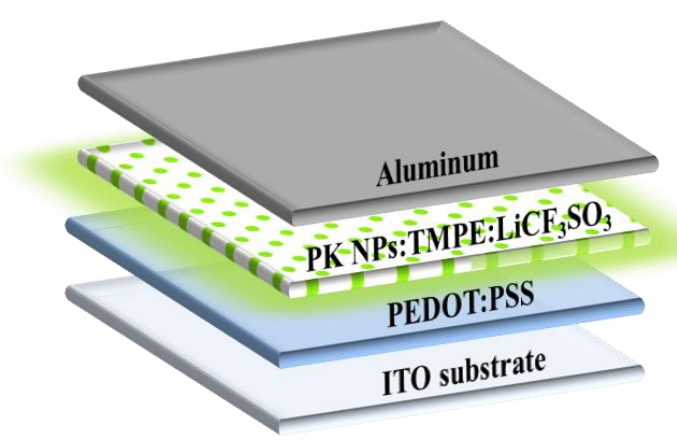

b)

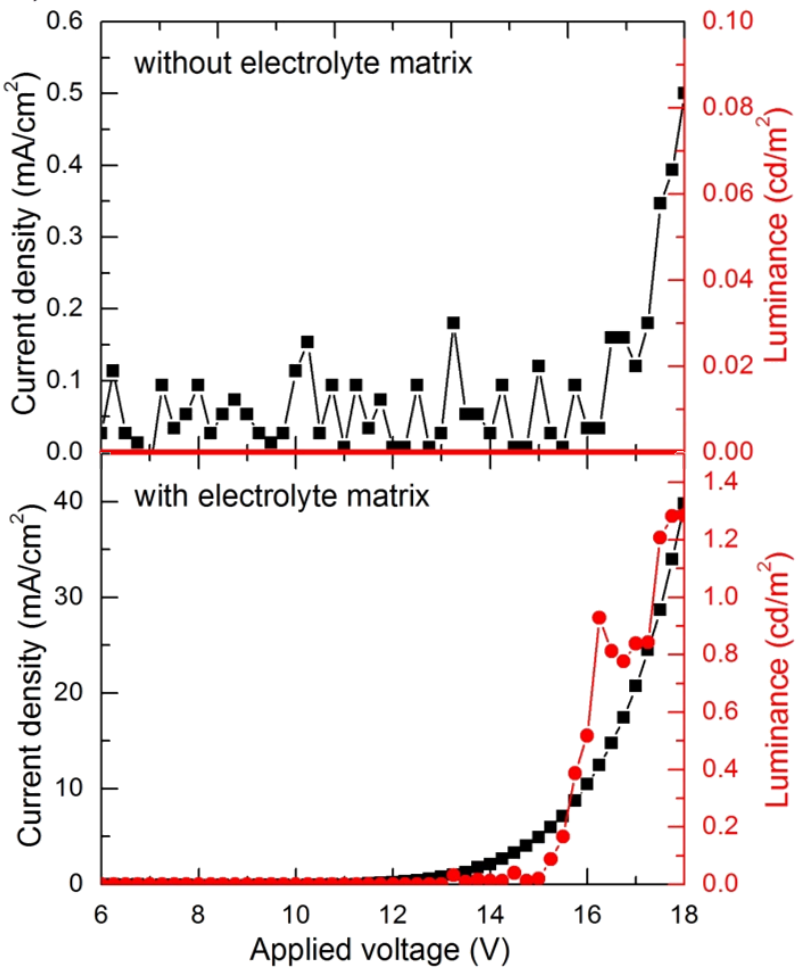

Figure 4. (a) Illustration of device architecture employed in this work. (b) Luminance (red circles) - current density (black squares) versus applied voltage of ITO/PEDOT:PSS (100 nm) $/ \mathrm{MAPbBr}_{3} \mathrm{NPs}$ without electrolyte matrix $(50-70 \mathrm{~nm})$ and with electrolyte matrix $(100-120 \mathrm{~nm})$ /Al $(90 \mathrm{~nm})$ devices.

The electroluminescence (EL) spectra of both perovskite NPs based LECs are shown in Figure 5. In general, 3D plots of the EL during the LIV assays show no change in both the shape and the maximum upon increasing the applied bias. In particular, the EL spectra of $\mathrm{MAPbBr}_{3}$ and $\mathrm{FAPbBr}_{3} \mathrm{NPs}$ based devices are centered at 550 and $560 \mathrm{~nm}$ with Commission Internationale d'Eclairage 1931 (CIE) chromaticity coordinates (x,y) of $(0.39,0.46)$ and $(0.45,0.50)$, respectively - Figure $5 \mathrm{~b}$ and $\mathrm{S} 11$. This represents a red-shift of 20-30 nm compared to the PL spectra of the active layer - Figures $2 \mathrm{~b}$ and $5 \mathrm{~b}$. This finding has been typically observed in LECs 
and it is ascribed to the stabilization of the excited state due to polarization under the applied high electric field during operating conditions. ${ }^{26}$
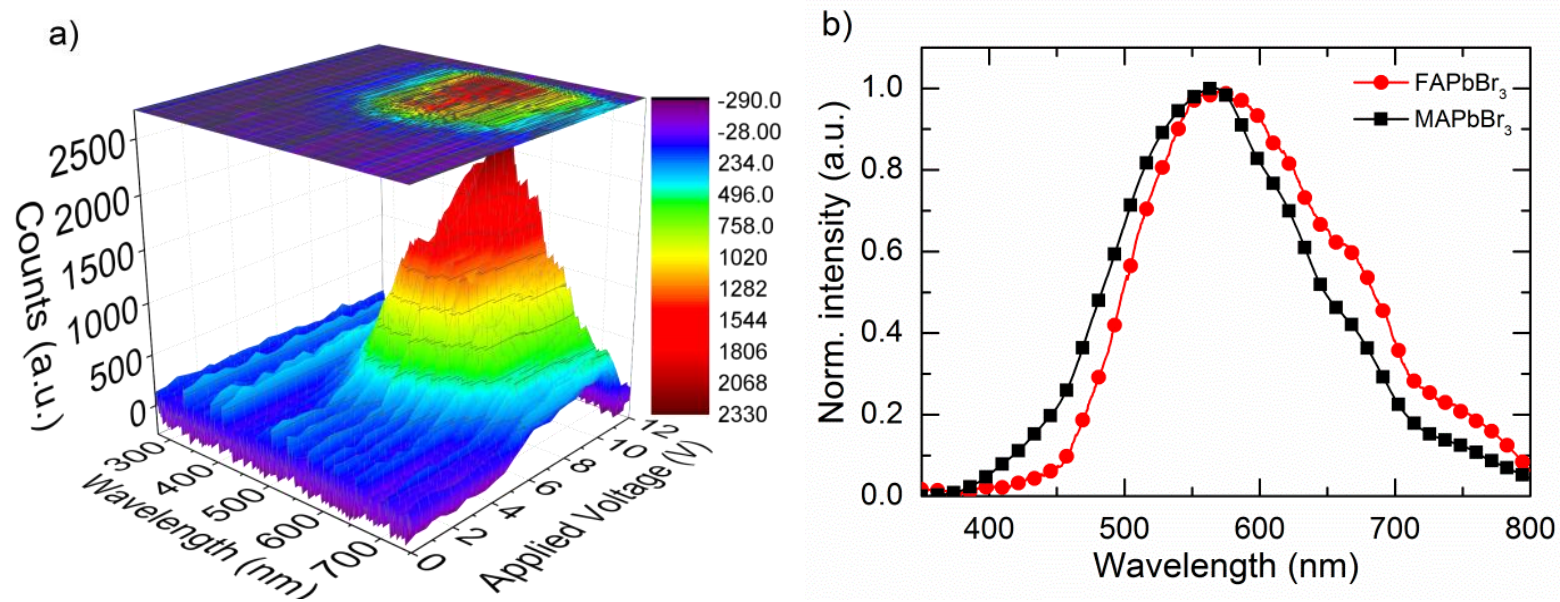

Figure 5. (a) $3 \mathrm{D}$ plot showing the EL spectra at different applied voltages during the LIV experiments of ITO/PEDOT:PSS/FAPbBr 3 NPs-electrolyte matrix (60-70 nm)/Al devices and (b) normalized EL spectra of ITO/PEDOT:PSS/Perovskite NP-electrolyte matrix (60-70nm)/Al devices at $10 \mathrm{~V}$.

The benefit of blending perovskite NPs with an ionic-based polyelectrolyte is clearly highlighted by both the strong reduction of the injection voltage and the stable EL features. Thus, we can postulate that perovskite NP-based devices show a common LEC behavior. ${ }^{1-4}$ For instance, upon application of a traditional constant voltage driving scheme - Figures 6a and S12a, the current density increases followed by a slow increase of the luminance, reaching values of 14 and $10 \mathrm{~mA} / \mathrm{cm}^{2}$ and 2 and $0.25 \mathrm{~cd} / \mathrm{m}^{2}$ for $\mathrm{MAPbBr}_{3}$ - and $\mathrm{FAPbBr}_{3}$-based devices. This behavior is ascribed to the slow redistribution of the mobile ions at the electrode interface together with a fast growth of the $\mathrm{p}$ - and n-doped regions, ${ }^{27-31}$ limiting the device performance. Recently, the application of a pulsed current driving scheme has been proposed to prevent the fast growth of the doped regions, while ensuring enough charge injection to provide an 
instantaneous emission..$^{1-2,32}$ In line with previous studies, upon application of a pulsed current scheme, initial high voltages were required to maintain the desired current density as the redistribution of ions has not been realized yet - see inset graphs in Figures $6 \mathrm{~b}$ and S12b. This allows both charge injection and a very slow redistribution of the mobile ions at the electrode interface. As such, the luminance is almost instantaneous and the average voltage decreases over the first minutes and remains constant, reaching maximum luminances of 1.8 and $0.8 \mathrm{~cd} / \mathrm{m}^{2}$ and efficiencies of 0.013 and $0.005 \mathrm{~cd} / \mathrm{A}$, as well as lifetimes of several hours for devices with $\mathrm{MAPbBr}_{3}$ and $\mathrm{FAPbBr}_{3} \mathrm{NPs}$, respectively. Since the average voltage profile remains practically constant, the reason for the loss in luminance might be ascribed to the intrinsic device operating mechanism. ${ }^{27-31}$ It is important to point out that, in good agreement with the PL features of the NPs, the $\mathrm{MAPbBr}_{3}$ NP-based devices outperform $\mathrm{FAPbBr}_{3} \mathrm{NP}$-based ones.
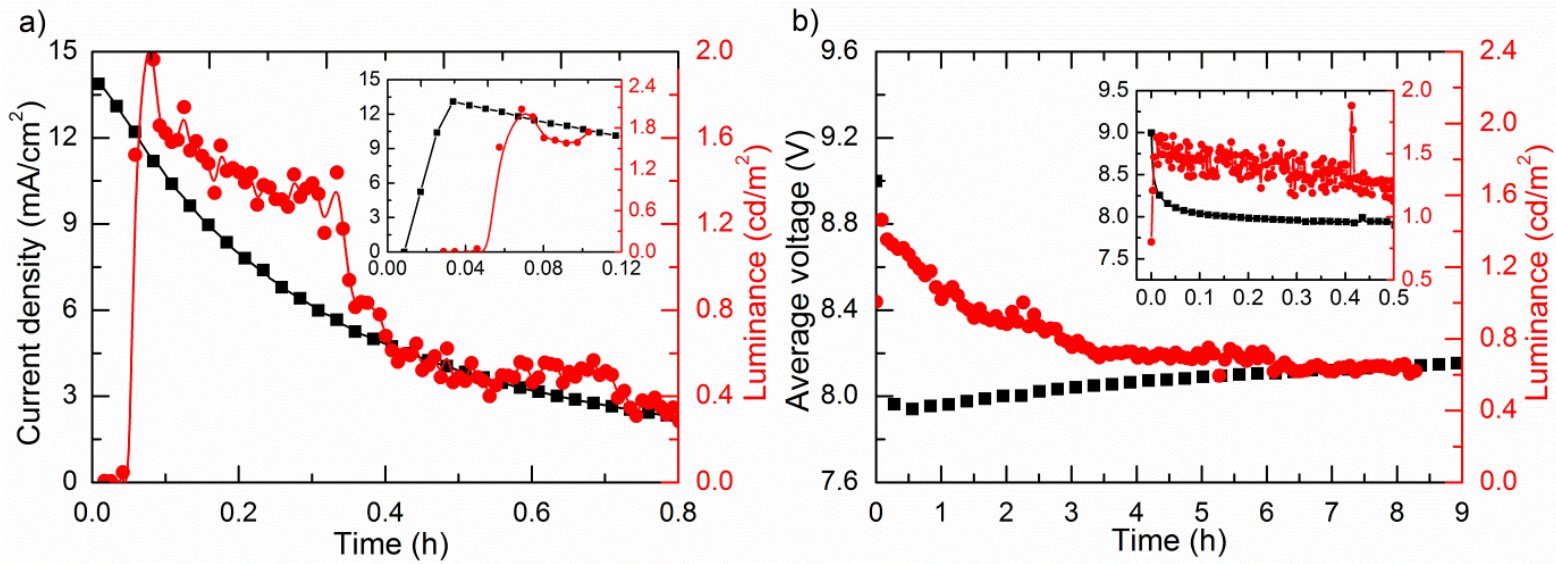

Figure 6. (a) Current density-luminance versus time of ITO/PEDOT:PSS/MAPbBr ${ }_{3} \mathrm{NP}-$ electrolyte matrix (100-120 nm)/Al driven by constant voltage of $14 \mathrm{~V}$ and (b) average voltageluminance versus time of the same device driven by a pulsed current using a block wave at a frequency of $1 \mathrm{kHz}$ with a duty cycle of $50 \%$ and an average current density of $13 \mathrm{~mA} / \mathrm{cm}^{2}$. The insets in both figures show the response of the device over first minutes. 
Finally, we have also evaluated the air stability of the devices stored under ambient conditions for a period of two months. Surprisingly, both the device and the photoluminescence features do not seem to be significantly affected as shown in Figures 7 and S13. In particular, the shape and maximum of the EL spectra remain unaltered, while the two month old devices show slightly reduced luminance and efficiency levels when compared to fresh devices. This is quite likely to the result of unbalanced electron-hole recombination due to an increased leakage current caused by the degradation of the active layer and/or accumulation of moisture over time when stored under ambient conditions.

a)

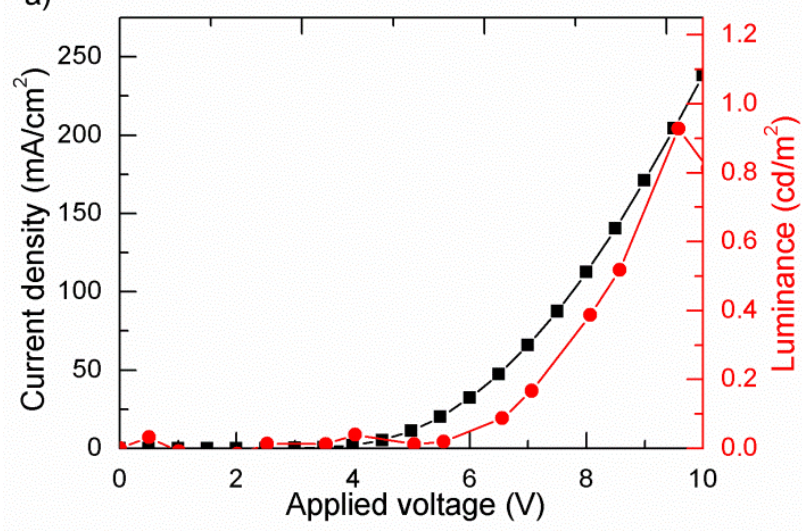

c)

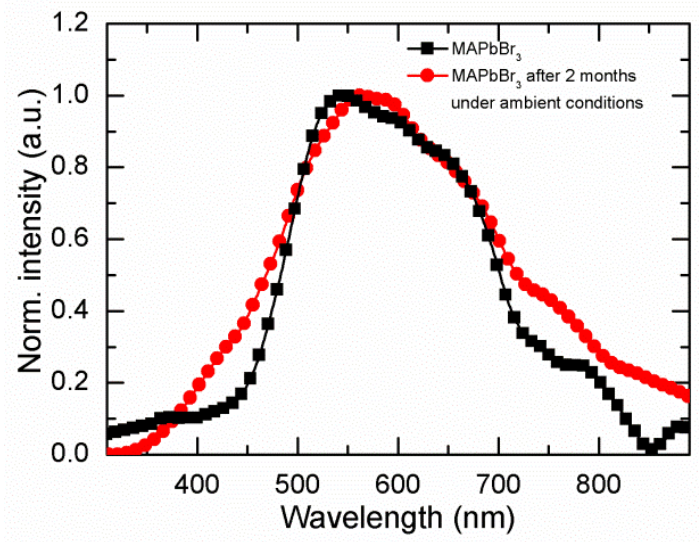

b)

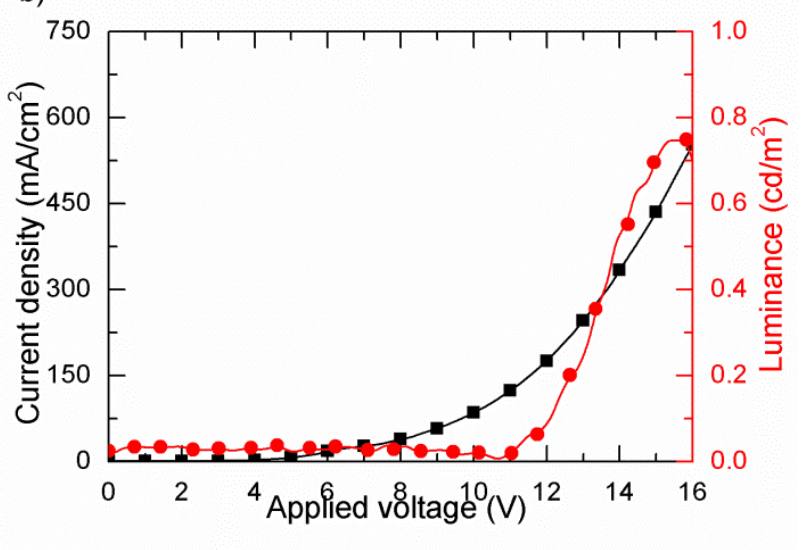

d)

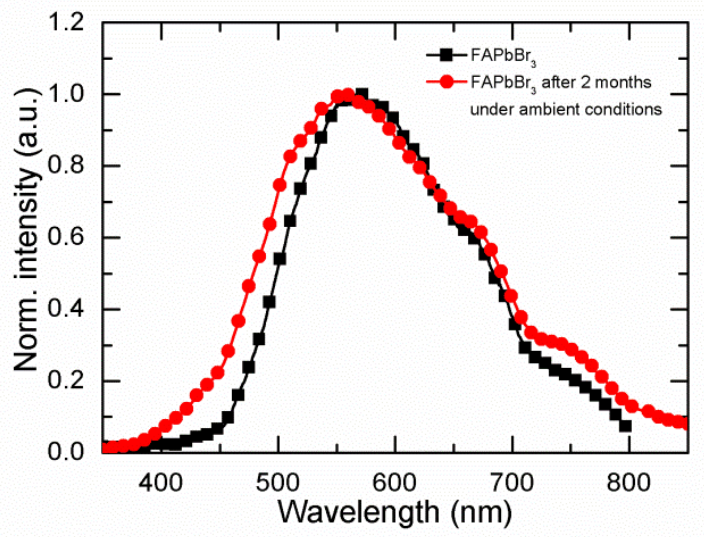

Figure 7. Luminance-current density versus applied voltage plot of ITO/PEDOT:PSS/FAPbBr 3 NP-electrolyte matrix $(60-70 \mathrm{~nm}) / \mathrm{Al}$ for fresh device (a) and same device kept under ambient 
conditions for two months (b). Electroluminescence spectra of both fresh and two month old MAPbBr 3 -based (c) and $\mathrm{FAPbBr}_{3}$-based (d) devices obtained at the maximum luminance level of their respective LIV assays.

\section{CONCLUSIONS}

In this work, we have demonstrated the implementation of perovskite NPs into light-emitting electrochemical cells for the first time. We have synthesized hybrid perovskite NPs with two different organic cations which were then incorporated into the final device via an up-scalable spray-coating technique. Exchanging the organic cation from MA to FA resulted in a 13 nm redshift of both the emission and absorption spectra. Our results show that FA-based NPs in solution exhibit six times slower radiative recombination rate which leads to a poorer PL quantum yield and, in turn, to a lower device performance. Additionally, we have investigated the origin of photoluminescence in these materials. We conclude that PL arises from bimolecular recombination of electrons in the conduction band and holes in the valence band. Moreover, devices prepared with the electrolyte matrix showed stable yellowish green luminance at low driving currents while those without this component did not show any EL features. Finally, we have demonstrated the stability of these devices and films stored under ambient conditions for over two months. Overall, this study opens up a new route to study organic-inorganic metal halide perovskite NPs and, additionally, opens a new avenue of research into the emerging field of LECs.

\section{ASSOCIATED CONTENT}

Additional figures, including XRD patterns, ${ }^{1} \mathrm{H}-\mathrm{NMR}$ spectra, TEM images, Tauc plots, PL and time-resolved PL spectra of NPs with electrolyte matrix, CIE 1931 color space chromaticity, 
LIV characterization of $\mathrm{FAPbBr}_{3} \mathrm{NPs}$. This material is available free of charge via the Internet at http://pubs.acs.org.

\section{AUTHOR INFORMATION}

\section{Corresponding Authors}

*E-mail: pablo.docampo@cup.lmu.de and ruben.costa@fau.de

\section{Author Contributions}

${ }^{\ddagger}$ These authors contributed equally to the work.

\section{ACKNOWLEDGMENTS}

The authors acknowledge funding from the Bavarian Ministry of the Environment, the Bavarian Network "Solar Technologies Go Hybrid", and the DFG Excellence Clusters 'Nanosystems Initiative Munich' (NIM) and 'Engineering of Advanced Materials' (EAM). RDC and MDW acknowledge the Fonds der Chemischen Industrie (FCI) in the Liebig grant framework. PD acknowledges support from the European Union through the award of a Marie Curie Intra-European Fellowship.

\section{REFERENCES}

(1) Costa, R. D.; Orti, E.; Bolink, H. J.; Monti, F.; Accorsi, G.; Armaroli, N. Luminescent Ionic Transition-Metal Complexes for Light-Emitting Electrochemical Cells. Angew. Chem. Int. Ed. 2012, 51, 8178-8211. 
(2) Meier, S. B.; Tordera, D.; Pertegás, A.; Roldán-Carmona, C.; Ortí, E.; Bolink, H. J. Light-Emitting Electrochemical Cells: Recent Progress and Future Prospects. Mater. Today 2014, 17, 217-223.

(3) Sandström, A.; Asadpoordarvish, A.; Enevold, J.; Edman, L. Light Emission: Spraying Light: Ambient-Air Fabrication of Large-Area Emissive Devices on Complex-Shaped Surfaces. Adv. Mater. 2014, 26, 4974-4974.

(4) YU ZhiBin, L. L., GAO HuiEr, PEI QiBing Polymer Light-Emitting Electrochemical Cells: Recent Developments to Stabilize the p-i-n Junction and Explore Novel Device Applications. Sci. China Chem. 2013, 56, 1075-1086.

(5) Tang, S.; Tan, W.-Y.; Zhu, X.-H.; Edman, L. Small-Molecule Light-Emitting Electrochemical Cells: Evidence for in situ Electrochemical Doping and Functional Operation. Chem. Commun. 2013, 49, 4926-4928.

(6) Pertegás, A.; Tordera, D.; Serrano-Pérez, J. J.; Ortí, E.; Bolink, H. J. Light-Emitting Electrochemical Cells Using Cyanine Dyes as the Active Components. J. Am. Chem. Soc. 2013, 135, 18008-18011.

(7) Bader, A. J. N.; Ilkevich, A. A.; Kosilkin, I. V.; Leger, J. M. Precise Color Tuning via Hybrid Light-Emitting Electrochemical Cells. Nano Lett. 2011, 11, 461-465.

(8) Qian, G.; Lin, Y.; Wantz, G.; Davis, A. R.; Carter, K. R.; Watkins, J. J. Saturated and Multi-Colored Electroluminescence from Quantum Dots Based Light Emitting Electrochemical Cells. Adv. Funct. Mater. 2014, 24, 4484-4490.

(9) Nozik, A. J. Quantum Dot Solar Cells. Physica E 2002, 14, 115-120. 
(10) Deschler, F.; Price, M.; Pathak, S.; Klintberg, L. E.; Jarausch, D.-D.; Higler, R.; Huettner, S.; Leijtens, T.; Stranks, S. D.; Snaith, H. J., et al. High Photoluminescence Efficiency and Optically Pumped Lasing in Solution-Processed Mixed Halide Perovskite Semiconductors. J. Phys. Chem. Lett. 2014, 5, 1421-1426.

(11) Stamplecoskie, K. G.; Manser, J. S.; Kamat, P. V. Dual Nature of the Excited State in Organic-Inorganic Lead Halide Perovskites. Energy Environ. Sci. 2015, 8, 208-215.

(12) Era, M.; Morimoto, S.; Tsutsui, T.; Saito, S. Organic-Inorganic Heterostructure Electroluminescent Device Using a Layered Perovskite Semiconductor $\left(\mathrm{C}_{6} \mathrm{H}_{5} \mathrm{C}_{2} \mathrm{H}_{4} \mathrm{NH}_{3}\right)_{2} \mathrm{PbI}_{4}$. Appl. Phys. Lett. 1994, 65, 676-678.

(13) Chondroudis, K.; Mitzi, D. B. Electroluminescence from an Organic-Inorganic Perovskite Incorporating a Quaterthiophene Dye within Lead Halide Perovskite Layers. Chem. Mater. 1999, 11, 3028-3030.

(14) Hattori, T.; Taira, T.; Era, M.; Tsutsui, T.; Saito, S. Highly Efficient Electroluminescence from a Heterostructure Device Combined with Emissive Layered-Perovskite and an ElectronTransporting Organic Compound. Chem. Phys. Lett. 1996, 254, 103-108.

(15) Era, M.; Morimoto, S.; Tsutsui, T.; Saito, S. Electroluminsecent Device Using 2Dimensional Semiconductor $\left(\mathrm{C}_{6} \mathrm{H}_{5} \mathrm{C}_{2} \mathrm{H}_{4} \mathrm{NH}_{3}\right)_{2} \mathrm{PbI}_{4}$ As an Emitter. Synth. Met. 1995, 71, 20132014.

(16) Tan, Z.-K.; Moghaddam, R. S.; Lai, M. L.; Docampo, P.; Higler, R.; Deschler, F.; Price, M.; Sadhanala, A.; Pazos, L. M.; Credgington, D., et al. Bright Light-Emitting Diodes Based On Organometal Halide Perovskite. Nat. Nanotechnol. 2014, 9, 687-692. 
(17) Kim, Y.-H.; Cho, H.; Heo, J. H.; Kim, T.-S.; Myoung, N.; Lee, C.-L.; Im, S. H.; Lee, T.W. Multicolored Organic/Inorganic Hybrid Perovskite Light-Emitting Diodes. Adv. Mater. 2014, $27,1248-1254$.

(18) Schmidt, L. C.; Pertegas, A.; Gonzalez-Carrero, S.; Malinkiewicz, O.; Agouram, S.; Minguez Espallargas, G.; Bolink, H. J.; Galian, R. E.; Perez-Prieto, J. Nontemplate Synthesis of $\mathrm{CH}_{3} \mathrm{NH}_{3} \mathrm{PbBr}_{3}$ Perovskite Nanoparticles. J. Am. Chem. Soc. 2014, 136, 850-853.

(19) Hanusch, F. C.; Wiesenmayer, E.; Mankel, E.; Binek, A.; Angloher, P.; Fraunhofer, C.; Giesbrecht, N.; Feckl, J. M.; Jaegermann, W.; Johrendt, D., et al. Efficient Planar Heterojunction Perovskite Solar Cells Based on Formamidinium Lead Bromide. J. Phys. Chem. Lett. 2014, 5, 2791-2795.

(20) Mitzi, D. B. Templating and Structural Engineering in Organic-Inorganic Perovskites. $J$. Chem. Soc., Dalton Trans. 2001, 1, 1-12.

(21) Eperon, G. E.; Stranks, S. D.; Menelaou, C.; Johnston, M. B.; Herz, L. M.; Snaith, H. J. Formamidinium Lead Trihalide: a Broadly Tunable Perovskite for Efficient Planar Heterojunction Solar Cells. Energy Environ. Sci. 2014, 7, 982-988.

(22) Wehrenfennig, C.; Liu, M.; Snaith, H. J.; Johnston, M. B.; Herz, L. M. Charge-Carrier Dynamics in Vapour-Deposited Films of the Organolead Halide Perovskite $\mathrm{CH}_{3} \mathrm{NH}_{3} \mathrm{PbI}_{3-\mathrm{x}} \mathrm{Cl}_{\mathrm{x}}$. Energy Environ. Sci. 2014, 7, 2269-2275.

(23) Stranks, S. D.; Burlakov, V. M.; Leijtens, T.; Ball, J. M.; Goriely, A.; Snaith, H. J. Recombination Kinetics in Organic-Inorganic Perovskites: Excitons, Free Charge, and Subgap States. Phys. Rev. Applied 2014, 2, 034007. 
(24) Vak, D.; Kim, S.-S.; Jo, J.; Oh, S.-H.; Na, S.-I.; Kim, J.; Kim, D.-Y. Fabrication of Organic Bulk Heterojunction Solar Cells by a Spray Deposition Method for Low-cost Power Generation. Appl. Phys. Lett. 2007, 91, 081102-081102-3.

(25) Green, R.; Morfa, A.; Ferguson, A. J.; Kopidakis, N.; Rumbles, G.; Shaheen, S. E. Performance of Bulk Heterojunction Photovoltaic Devices Prepared by Airbrush Spray Deposition. Appl. Phys. Lett. 2008, 92, 033301-033301-3.

(26) Wang, Y.-M.; Teng, F.; Hou, Y.-B.; Xu, Z.; Wang, Y.-S.; Fu, W.-F. Copper(I) Complex Employed in Organic Light-Emitting Electrochemical Cells: Device and Spectra Shift. Appl. Phys. Lett. 2005, 87, 233512-233512-3.

(27) Matyba, P.; Maturova, K.; Kemerink, M.; Robinson, N. D.; Edman, L. The Dynamic Organic p-n Junction. Nat. Mater. 2009, 8, 672-676.

(28) Slinker, J. D.; DeFranco, J. A.; Jaquith, M. J.; Silveira, W. R.; Zhong, Y.-W.; MoranMirabal, J. M.; Craighead, H. G.; Abruna, H. D.; Marohn, J. A.; Malliaras, G. G. Direct Measurement of the Electric-Field Distribution in a Light-Emitting Electrochemical Cell. Nat. Mater. 2007, 6, 894-899.

(29) Malliaras, G. G.; Slinker, J. D.; DeFranco, J. A.; Jaquith, M. J.; Silveira, W. R.; Zhong, Y.-W.; Moran-Mirabal, J. M.; Craighead, H. G.; Abruna, H. D.; Marohn, J. A. Operating Mechanism of Light-Emitting Electrochemical Cells. Nat. Mater. 2008, 7, 168-168.

(30) van Reenen, S.; Akatsuka, T.; Tordera, D.; Kemerink, M.; Bolink, H. J. Universal Transients in Polymer and Ionic Transition Metal Complex Light-Emitting Electrochemical Cells. J. Am. Chem. Soc. 2012, 135, 886-891. 
(31) Meier, S. B.; van Reenen, S.; Lefevre, B.; Hartmann, D.; Bolink, H. J.; Winnacker, A.;

Sarfert, W.; Kemerink, M. Dynamic Doping in Planar Ionic Transition Metal Complex-Based Light-Emitting Electrochemical Cells. Adv. Funct. Mater. 2013, 23, 3531-3538.

(32) Tordera, D.; Meier, S.; Lenes, M.; Costa, R. D.; Ortí, E.; Sarfert, W.; Bolink, H. J. Simple, Fast, Bright, and Stable Light Sources. Adv. Mater. 2012, 24, 897-900.

\section{TOC GRAPHICS}

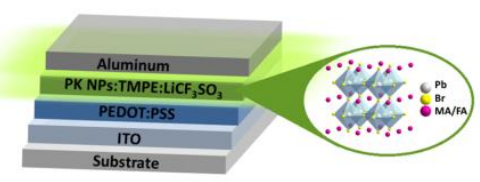

